\title{
Riesige Mehrkosten
}

\section{"Versorgungslandschaft Diabetes" in Nöten}

\begin{abstract}
Jährlich erkranken in Deutschland 270000 Personen an Diabetes, fast jeder dritte 70-Jährige ist betroffen. Stark betroffen sind auch die Kassen der Krankenkassen: 1,8 Milliarden Mehrkosten resultieren aus der jährlichen Zunahme, ohne Einbeziehung indirekter Kosten. Eine gigantische Herausforderung für die Versorgungsstrukturen, Alarmrufe sind schon der Normalfall geworden.
\end{abstract}

I— DDG-Präsident Prof. Stefan Matthaei fügt diesem aktualisierten Horrorszenarium noch die Botschaft hinzu, dass es dramatische Unterschiede in der Diabetes-Inzidenz in Deutschland gibt: In Sachsen-Anhalt 12\%, in Bayern 5,8\%, wobei es eine sehr enge Korrelation von Arbeitslosigkeit und Diabetes zu berücksichtigen gilt. Liegt die Arbeitslosigkeit im Wohnumfeld bei $20 \%$ und darüber, steigt die Diabetes-Häufigkeit auf Spitzenwerte über $11 \%$. Zwischen 5 und $8,9 \%$ sinkt die Diabetes-Inzidenz auf 2,5\%.

Derzeit schätzt man die Gesamtzahl der Diabetiker in Deutschland auf etwa 7 Millionen, die Kosten in Höhe von jährlich rund 25 Milliarden verursachen.

\section{Klare Therapieziele,} dürftige Umsetzung Die Therapieziele, so Matthaei, sind klar und evidenzbasiert, "die Therapiezielerreichung schlecht bis mittelmäßig". Nach Daten aus 2009 erreichen 60\% der Typ-2-Diabetiker ein $\mathrm{HbA}_{1 \mathrm{c}}$, die Blutdruckziele von 130/80 mmHg knapp 30\%, die LDL-Ziele ebenso wenige.

Als Ansätze für eine Optimierung der Versorgung sieht Matthaei eine Verbesserung der Abstimmung in allen Versorgungsebenen: Hausärzte betreuen ca. 90\% der Diabetiker, dann kommen die DiabetesSchwerpunktpraxen und schließlich die Diabetes-Kliniken. Ein Projekt "Versorgungslandschaft Diabetes" soll das nun ändern, es sollen sich bislang daran 5 Fachgesellschaften bzw. Verbände, auch der Deutsche Hausarztverband, und Kassen beteiligen.

\section{Individualisierte Behandlungsziele - ein Paradigmenwechsel}

Die Behandlungsziele wurden in den letzten Jahren mehrfach nachjustiert, die strenge Einstellung sowie des $\mathrm{HbA}_{1 c}$ wie auch der Blutdruckzielwerte wurden gelockert, vor allem für den älteren Diabetiker. Man kann auch von einem Paradigmenwechsel sprechen, das dokumentieren jedenfalls die neuen, erst Mitte April 2012 publizierten gemeinsamen Leitlinien der Amerikanischen Diabetesgesellschaft (ADA) und der Europäischen Diabetesgesellschaft (EASD) zurTherapie des Typ-2-Diabetes (T2D).

Im Unterschied zur bisherigen Leitlinie der beiden Gesellschaften und auch zu vielen anderen Leitlinien von Fachgesell-

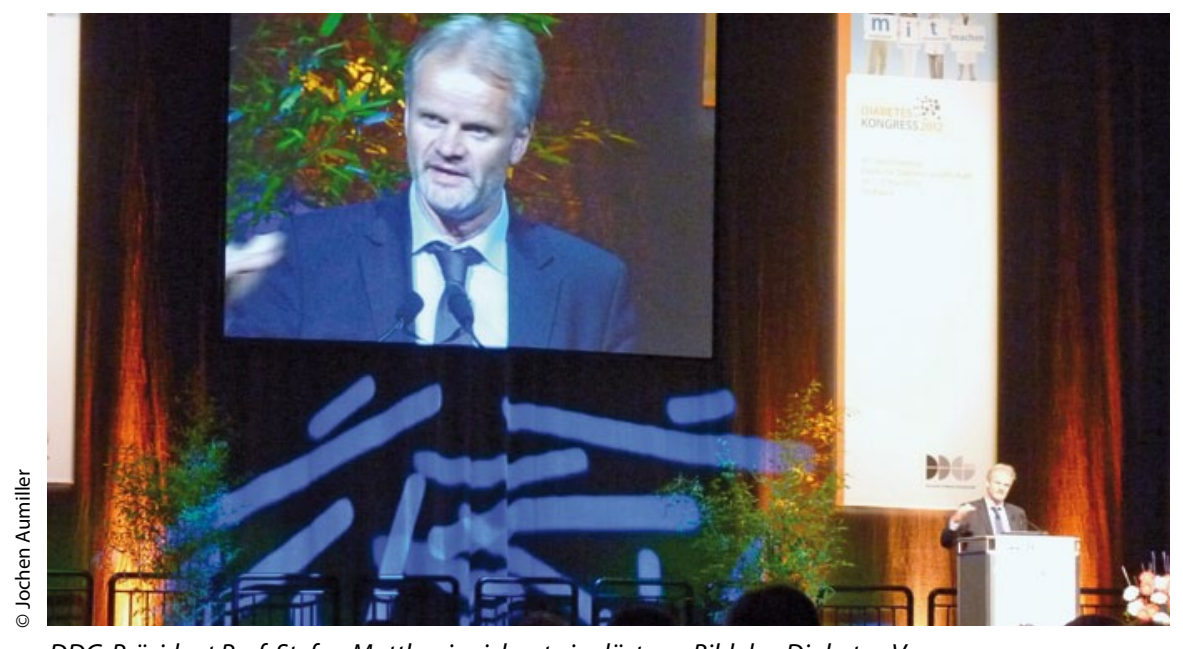

DDG-Präsident Prof. Stefan Matthaei zeichnet ein düsteres Bild der Diabetes-Versorgung.

schaften gibt diese deutlich weniger genaue Vorschriften und Empfehlungen zu den Therapiezielen und Medikamenten in Form von Algorithmen. Vielmehr rückt die neue Leitlinie den individuellen Patienten in den Mittelpunkt.

Das Therapieziel, definiert als Zielwert des "Langzeit-Blutzuckers" $\mathrm{HbA}_{1 c}$, legt die Leitlinie nicht mehr mit einer starren Zahl, zum Beispiel 6,5 Prozent oder 7 Prozent, fest. Stattdessen soll es für jeden einzelnen Patienten individuell bestimmt werden: Ein älterer Mensch mit Herz-Kreislauf-Erkrankungen etwa und lange bestehendem Diabetes wird weniger streng einzustellen sein als ein junger Diabetespatient.

Als erste Therapiemaßnahme nach der Diagnose eines T2D empfiehlt die Leitlinie erneut allein Lebensstiländerungen wie Ernährungsumstellung und Bewegung, verbunden mit intensiver Schulung. Eine sofortige Gabe von Tabletten sieht sie dagegen nicht vor. Erst wenn sich die „nicht-pharmakologische" Therapie als unwirksam erweise, seien Tabletten indiziert. Hier stehe an erster Stelle nach wie vor möglichst Metformin, kommentiert Professor Helmut Schatz, Pressesprecher der Deutschen Gesellschaft für Endokrinologie: „Was im nächsten Schritt gegeben werden soll, ist derzeit schwer zu entscheiden, da es für die Kombinationstherapien kaum,Outcome-Studien' gibt."

Die Leitlinie empfiehlt deshalb, die Wahl des zweiten Medikaments entsprechend den individuellen Merkmalen des Patienten zu treffen - etwa seinem Gewicht - und dies gemeinsam mit ihm festzulegen. Dies kann ein zweites Präparat in Tablettenform sein, aber auch die Injektion der neueren „GLP-1Analoga“ oder Insulin. Wolle ein Patient keine Injektionen, sollte der Arzt davon möglichst absehen. Nach längerer Krankheitsdauer wird Insulin allerdings unumgänglich.

Neue Leitlinien der ADA und EASD: Inzucchi SE, et al. Management of hyperglycaemia in type 2 diabetes: a patient-centered approach. Position statement of the American Diabetes Association (ADA) and the European Association for the Study of Diabetes (EASD). Diabetologia. 2012 Jun;55(6):1577-96.

Jochen Aumiller I 